\title{
Long Non-coding RNA GAS5 Worsens Coronary Atherosclerosis Through MicroRNA-194-3p/TXNIP Axis
}

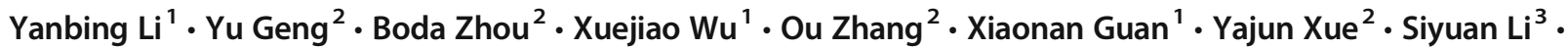 \\ Xianjing Zhuang ${ }^{3} \cdot$ Jie Zhou ${ }^{2} \cdot$ Meng Chang $^{3} \cdot$ Guobin Miao $^{2}$ (D) - Lizhong Wang ${ }^{4}$
}

Received: 29 September 2020 / Accepted: 15 February 2021 / Published online: 27 February 2021

(C) The Author(s) 2021

\begin{abstract}
It is formerly conducted that long non-coding RNA growth arrest-specific 5 (GAS5) is involved in the process of coronary atherosclerosis (AS). The regulatory effects of GAS5 on the microRNA (miR)-194-3p/thioredoxin-interacting protein (TXNIP) axis in AS have been insufficiently explored yet. Thereafter, this work is started from GAS5/miR-194-3p/TXNIP axis in AS. AS rats were modeled to obtain their coronary vascular tissues and endothelial cells (ECs), in which GAS5, miR-194-3p, and TXNIP expression were tested. ECs were identified by immunohistochemistry. The mechanism among GAS5, miR-194-3p, and TXNIP was determined. ECs were transfected with inhibited GAS5 or overexpressed miR-194-3p to decipher their functions in proliferation and apoptosis of ECs in AS. Raised GAS5 and TXNIP and degraded miR-194-3p expression levels exhibited in AS. GAS5 bound to miR-194-3p while miR-194-3p targeted TXNIP. Depleting GAS5 or restoring miR-194-3p enhanced proliferation and depressed apoptosis of ECs in AS. This work clearly manifests that inhibited GAS5 facilitates the growth of ECs through miR-194-3p-targeted TXNIP in AS, consolidating the basal reference to the curing for AS.
\end{abstract}

Keywords Coronary atherosclerosis - Long non-coding RNA growth arrest-specific 5 - MicroRNA-194-3p · Thioredoxin-interacting protein $\cdot$ Endothelial cells $\cdot$ Proliferation $\cdot$ Apoptosis

\section{Introduction}

Atherosclerosis (AS) is defined as a chronic inflammatory disease within the arterial wall, consequently resulting in peripheral artery disease, myocardial infarction, and coronary artery disease [1]. Pathologically, AS is mainly evoked from dyslipidemia, insulin resistance, hyperglycemia, oxidative

Yanbing Li and Yu Geng are co-first authors.

Guobin Miao

miaoguobin7723@163.com

$\triangle$ Lizhong Wang

wanglizhong9958@163.com

1 Department of Cardiology, Beijing Chaoyang Hospital, Capital Medical University, Beijing 100043, China

2 Department of Cardiology, Beijing Tsinghua Changgung Hospital, Tsinghua University, No. 168 Litang Road, Changping District, Beijing 102218, China

3 School of Clinical Medicine, Tsinghua University, Beijing, China

4 Cardiac Rehabilitation Center, Beijing Rehabilitation Hospital, Capital Medical University, Xixiazhuang, Badachu Road, Shijingshan Distract, Beijing 100144, China stress, and inflammation [2]. Actually, various cells including endothelial cells (ECs) and smooth muscle cells are substantially implied to connect with the generation of atherosclerotic plaques [3]. Specifically, the dysfunction of ECs comprises of non-adaptive changes in functional phenotypes, which are indicative in regulating thrombosis, hemostasis, local vascular tone and redox balance, and internal acute and chronic inflammatory responses [4]. Thereby, targeting ECs to improve their functions is potentially essential to manage AS.

It is instructive that long non-coding RNA growth arrestspecific 5 (GAS5) plays a role in inflammatory diseases, apoptosis of vascular ECs, and AS [5]. Besides that, GAS5 has been implied in the progression of AS through acting in the field of cholesterol reverse-transport and intracellular lipid accumulation [6]. Also, it is specifically elucidated that GAS5 influences autophagy dysfunction of ECs in AS [7]. Moreover, Shen S et al. have found that GAS5 performs crucially in the process of lipid metabolism and inflammation in AS via modulating microRNA (miR)-135a [8], indicating the co-expression of $\operatorname{lncRNAs}$ and miRNAs in AS. MiRNAs can alter atherosclerotic plaque progression/regression balance through the modification of signaling and lipid homeostasis pathways [9]. Pertaining to the group of miRNAs, miR-194 is 
reported to restrict inflammatory response and impair the permeability of human dermal microvascular ECs [10]. Functionally, miR-194 is involved in the transition of astrocytes into ECs and participates in gene regulation of AS [11]. Thioredoxin-interacting protein (TXNIP), an endogenous inhibitor of thioredoxin, is documented to regulate diabetesaccelerated AS [12]. Furthermore, knocking down TXNIP functions to reduce the atherosclerotic lesion, implying its therapeutic potential in AS and inflammatory diseases [13]. Serving as the target gene of miR-20a, TXNIP can mediate inflammation in human aortic ECs, thereby to prevent the development of AS [14]. Concerning to that, it is a significant issue needed to be solved that whether GAS5 may exert as the regulator of miR-194-3p, which further targets the potential downstream gene TXNIP to affect AS. Thus, this work was initiated to explain how GAS5/miR-194-3p/TXNIP axis functioned in the progression of AS.

\section{Methods and Materials}

\section{Ethics Statement}

All animal experiments were executed with reference to the Guidelines for the Care and Use of Laboratory Animals (National Academy of Sciences Press, revised in 2010). The experimental operation was endorsed by the ethics committee of Beijing Tsinghua Changgung Hospital (ethical number: 201920629).

\section{Experimental Animals}

Male Sprague Dawley rats (6 weeks old) of specific pathogenfree grade (Beijing Vital River Laboratory Animal Technology Co., Ltd., Beijing, China) were adaptively reared for 1 week (with sufficient food and water, $20-25^{\circ} \mathrm{C}$, and relative humidity of $45-70 \%$ ).

\section{AS Rat Modeling and Treatment}

AS rats were raised for a week and injected with vitamin $D_{3}$ (GenePharma, Shanghai, China), with a total dose of 70,000 $\mathrm{U} / \mathrm{kg}$ for 3 days. Then, the rats were fed a high-fat diet containing $4 \%$ cholesterol, $5 \%$ glucose, $10 \%$ lard, and $82 \%$ normal diet for 21 days. The rats in the control group were fed a normal diet and intraperitoneally injected with physiological saline once a month. The biochemical changes of blood lipid were detected by an automatic biochemical analyzer after 12 weeks [15]. Rats were grouped as follows: control group (rats fed with standard food and drinking water), AS group (AS rats fed with high-fat diet and intraperitoneally injected with vitamin $\mathrm{D}_{3}$ ), sh-negative control (NC) group (AS rats fed with high-fat diet and intraperitoneally injected with vitamin $\mathrm{D}_{3}$, and injected with sh-GAS5 NC), sh-GAS5 group (AS rats fed with high-fat diet and intraperitoneally injected vitamin $D_{3}$, and injected with sh-GAS5), agomir NC group (AS rats fed with high-fat diet and intraperitoneally injected vitamin $\mathrm{D}_{3}$, and injected with miR-194-3p agomir NC), and miR-194-3p agomir group (AS rats fed with high-fat diet and intraperitoneally injected with vitamin $D_{3}$, and injected with miR-194-3p agomir). The transfection plasmids $(10 \mu \mathrm{L}$, all from Huibaibio, Shenyang, China) were injected into rats $(n=12$ / group) through the caudal vein at 21 days post modeling, and the needle was kept in the vein for $1 \mathrm{~min}$. Since that, the rats were continuously fed a high-fat diet [15].

\section{Specimen Collection}

At 16 weeks post injection, all rats were euthanized to obtain coronary tissues. ECs were collected as previously described [16]. Coronary tissues were detached in $0.1 \%$ collagenase to isolate ECs and centrifuged at $1200 \mathrm{rpm}$ to remove the supernatant. ECs were preserved at $-80^{\circ} \mathrm{C}$ and identified by immunohistochemistry testing factor VIII-related antigen von Willebrand factor (vWF) [15].

\section{Hematoxylin-Eosin Staining}

Coronary tissues were fixed in $10 \%$ formalin (NanJing JianCheng Bioengineering Institute, Nanjing, China) for $24 \mathrm{~h}$, followed by dehydration with ethanol (Sigma-Aldrich, St. Louis, Missouri, USA), clearance with xylene, dewaxing, and embedment in paraffin. After that, the paraffin-embedded block was dewaxed and stained with hematoxylin staining solution. Next, the tissues were differentiated by $1 \%$ acidic alcohol, immersed in eosin solution, and dehydrated in ethanol. After clearance with xylene twice $(10 \mathrm{~min})$, the tissues were covered with Acacia senegal and observed under a CX-31 optical microscope (Olympus, Tokyo, Japan). An LM1235 ultra-thin semi-automatic slicer (Leica, Wetzlar, Germany) was utilized for sectioning while a KD-BM machine for tissue embedment (Kedee, Zhejiang, China).

\section{Transferase-Mediated Deoxyuridine Triphosphate- Biotin Nick End Labeling Staining}

Transferase-mediated deoxyuridine triphosphate-biotin nick end labeling (TUNEL) staining was conducted with an in situ apoptosis detection kit (11684795910, Basel Roche, Switzerland). Paraffin-embedded sections were dewaxed and dehydrated, and treated with $20 \mathrm{mg} / \mathrm{mL}$ proteinase $\mathrm{K}$ and $3 \%$ $\mathrm{H}_{2} \mathrm{O}_{2}$ for $30 \mathrm{~min}$. Then, the sections were soaked in $1 \%$ citric acid solution $(0.1 \%$ Triton X-100) and supplemented with 50 $\mathrm{mL}$ TUNEL reaction mixture. The sections were treated with horseradish peroxidase-labeled goat anti-rabbit antibody and stained with diaminobenzidine (DAB). A color image 
analyzer (BI-2000) and a high-power microscope were applied for image analysis. In 5 randomly selected fields, the number of apoptotic cells was counted [17].

\section{Immunohistochemistry}

Coronary vascular tissues were fixed, sectioned, and sealed. After that, tissues were reacted with the primary antibody vascular endothelial growth factor (VEGF, 1:100, sc-7269, Santa Cruz Biotechnology, Santa Cruz, CA, USA) and with goat anti-rabbit secondary antibody immunoglobulin $\mathrm{G}$ (IgG, ab150077, 1:200, Abcam, MA, USA) in phosphate-buffered saline (PBS). Then, the tissues were kept in streptavidin biotin-peroxidase complex (Boster Biological Technology Co. Ltd., Hubei, China), stained with DAB (Boster), and observed by a microscope (Leica) [18].

Immunohistochemistry was applied to determine vWF in ECs. The collected cells were placed in $4 \%$ paraformaldehyde, frozen in $30 \%$ sucrose PBS, and sectioned into $30 \mu \mathrm{m}$ by a cryostat. Subsequently, cells were reacted with $0.3 \% \mathrm{H}_{2} \mathrm{O}_{2}$ or methanol and sealed with 10\% PBS. With En Vision two-step method, cells were probed with rabbit anti-human factor VIIrelated antigen polyclonal antibody (Neomarkers, CA, USA) and with anti-rabbit/mouse universal immunohistochemical kit (DAKO, MI, USA). Followed by that, cells were developed by $\mathrm{DAB}$, counterstained by hematoxylin solution, treated with ammonia, and observed under an optical microscope (Olympus).

\section{Cell Culture and Transfection}

ECs from rats in the control group and AS group were cultured in Dulbecco's modified Eagle's medium//high glucose (SH30022.01, Gibco, CA, USA) supplemented with $10 \%$ fetal bovine serum (HyCLone, UT, USA). Detached by trypsin (825M042, 1:250, Solarbio, China), ECs were passaged. Through restriction enzyme digestion and sequencing, miR194-3p agomir, miR-194-3p agomir NC, sh-NC, or sh-GAS5 were synthesized and packaged in liposomes. ECs at passage 3 with $80 \%$ confluence were transfected with the diluted liposomes and cultured for $24 \mathrm{~h} \mathrm{[16]}$. The cell groups are as follows: control group (ECs from rats in the control group), AS group (ECs from rats in the AS group), sh-NC group (ECs from rats in the AS group were transfected with sh-GAS5 NC), sh-GAS5 group (ECs from rats in the AS group were transfected with sh-GAS5), agomir NC group (ECs from rats in the AS group were transfected with miR-194-3p agomir NC), and miR-194-3p agomir group (ECs from rats in the AS group were transfected with miR-194-3p agomir).

\section{Reverse Transcription Quantitative Polymerase Chain Reaction}

Total RNA was extracted from coronary vascular tissues and ECs via Trizol reagent (ThermoFisher, Waltham, MA, USA) and then processed with reverse transcription via a cDNA transcription kit (ThermoFisher). U6 served as the internal control for GAS5 while glyceraldehyde-3-phosphate dehydrogenase served as the internal control for miR-194-3p and TXNIP. A SYBR-Green RT-PCR kit (Takara, Tokyo, Japan) was applied to test gene expression levels, which were further calculated by $2^{-\Delta \Delta \mathrm{Ct}}$ method $[19,20]$. Primers were listed in Table 1.

\section{Western Blot Assay}

Total protein was extracted from tissues and cells with radioimmunoprecipitation assay buffer, of which the protein concentration was measured by a bicinchoninic acid protein kit (ThermoFisher Scientific). Separated by $10 \%$ sodium dodecyl sulfate polyacrylamide gel electrophoresis, the protein was placed into a $0.45-\mu \mathrm{M}$ polyvinylidene fluoride membrane (Millipore, Billerica, MA, USA) and probed with the specific antibodies. Protein bands were developed by enhanced chemiluminescence and photographed by an Image Quant LAS 4000C gel imager (General Electric Company, Boston, MA, USA). Primary antibodies are as follows: TXNIP $(1: 1000$, ab188865, Abcam), VEGF (1:200, sc-7269, Santa Cruz Biotechnology), and vWF (1:1000, A0082, DAKO). Secondary antibody is anti-rabbit $\operatorname{IgG}(7074,1: 2000$, Cell Signaling Technology) [21]. Glyceraldehyde-3-phosphate dehydrogenase (1:2500, ab9485, Abcam) was used as the internal control.

Table 1 Primer sequences

\begin{tabular}{lll}
\hline Primers & Forward $\left(5^{\prime}-3^{\prime}\right)$ & Reverse $\left(5^{\prime}-3^{\prime}\right)$ \\
\hline GAS5 & AGCTGGAAGTTGAAATG & CAAGCCGACTCTCCATA \\
miR-194-3p & CTCGCTTCGGCAGCACA & AACGCTTCACGAATTTGCGT \\
TXNIP & ACTCCTCAAGATGGGTGGCAATC & ACATCCACCCAGCAAACACTCCT \\
U6 & CTCGCTTCGGCAGCACA & AACGCTTCACGAATTTGCGT \\
GAPDH & TGTGATGGGTGTGAACCACG & GAGCCCTTCCACAATGCCAA \\
& AGAA & AGTT
\end{tabular}

GAS5 long non-coding RNA growth arrest-specific 5; miR-194-3p microRNA-194-3p; TXNIP thioredoxininteracting protein; $G A P D H$ glyceraldehyde-3-phosphate dehydrogenase 
A

B
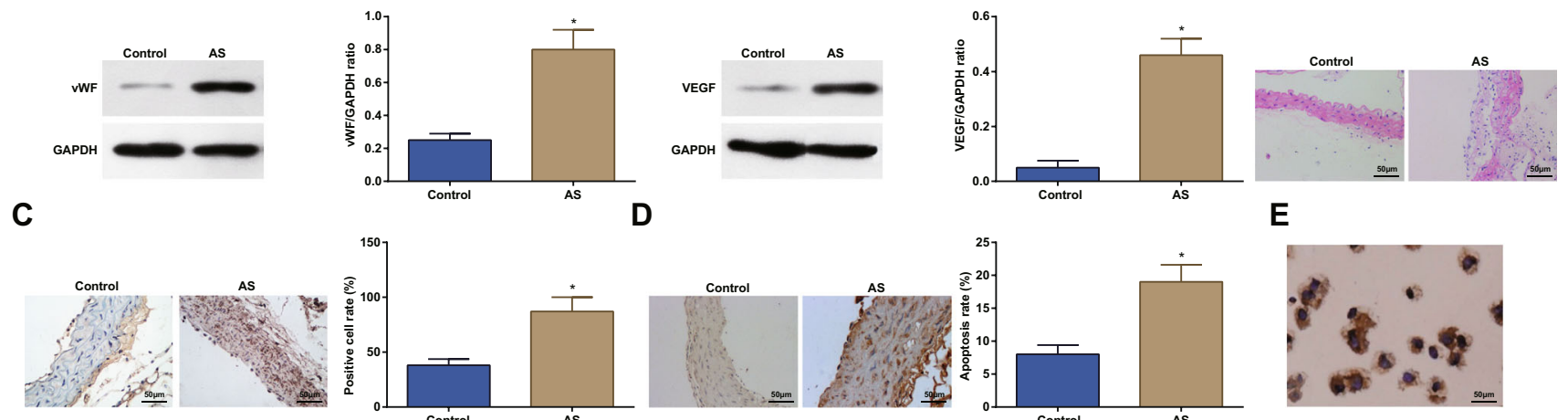

D
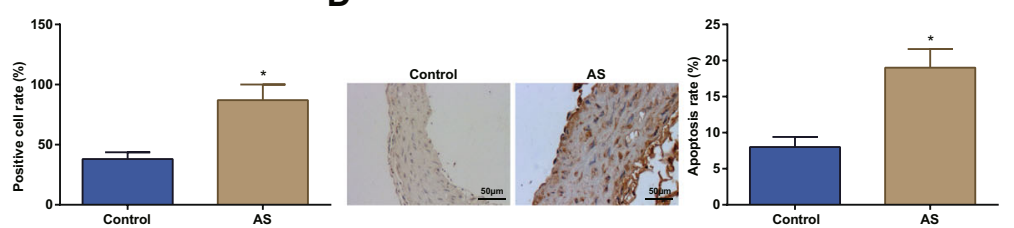

E

$\mathbf{F}$

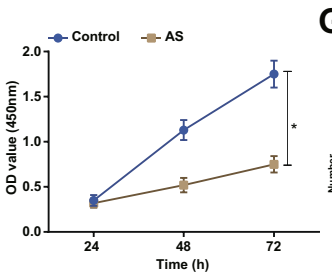

G
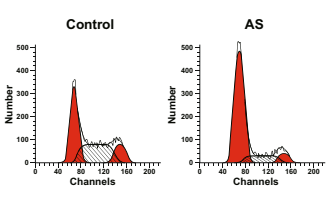

H

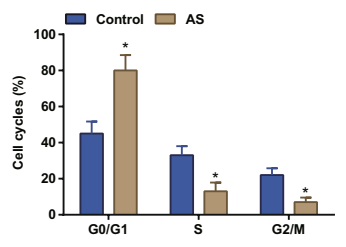

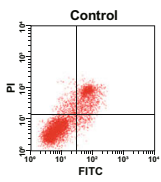
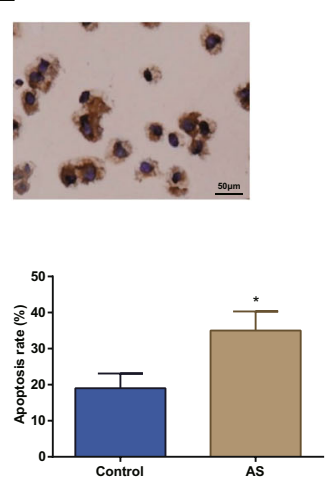

Fig. 1 Increased vWF and VEGF, inhibited proliferation of ECs, and promoted apoptosis are present in AS. a Western blot assay detected vWF and VEGF protein expression in ECs. b H\&E staining observed the morphology of ECs in coronary vascular tissues. c Immunohistochemistry detected VEGF expression in ECs. d TUNEL staining detected the apoptosis of ECs. e Immunohistochemistry identified ECs. f CCK-8 assay detected the proliferation of ECs. $\mathbf{g}$ and h Flow cytometry detected cell cycle distribution and apoptosis of ECs. * $P<0.05$ compared with the control group; the measurement data were expressed as the mean \pm standard deviation. Paired $t$ test was applied for discrepancy between the two groups. $N=3$

\section{Cell Counting Kit-8 Assay}

ECs were cultured under standard conditions to $70 \%$ confluence and seeded in 96-well plates at $1 \times 10^{4}$ cells/well. Cell proliferation was evaluated by a cell counting kit (CCK)-8 kit (Sigma, Tokyo, Japan). Optical density values were read at $450 \mathrm{~nm}$ [17].

\section{RNA Immunoprecipitation Assay}

A Magna RNA immunoprecipitation (RIP) kit (Millipore Corporation, MA, USA) was used in RIP assay by following the protocols. ECs with $85 \%$ confluence were lysed in complete RIP lysis buffer and incubated with anti-AGO2 (ab32381) or rabbit IgG (ab172730, both from Abcam). The purified RNA was detected by reverse transcription quantitative polymerase chain reaction (RT-qPCR) [22].

\section{Dual Luciferase Reporter Gene Assay}

The binding site of miR-194-3p with GAS5 or TXNIP was predicted by Jefferson website (https://cm.jefferson.edu/ rna22/Precomputed/). The 3'-untranslated region (UTR) sequences of GAS5 and TXNIP were synthesized, followed by digestion, purification, ligation with pGL3-control vector, transformation, and sequencing. GAS5-wild type (WT) 3'UTR and TXNIP-WT 3'-UTR were mutated to produce
GAS5-mutant (MUT) 3'-UTR and TXNIP-MUT 3'-UTR. Following the instructions of X-tremegeneHP Transfection Reagent (Roche), GAS5-WT/GAS5-MUT, TXNIP-WT/ TXNIP-MUT, and miR-194-3p-agomir/agomir-NC were added into opti-minimum essential medium and transfected into ECs. After $48 \mathrm{~h}$ of culture, the luciferase activity was measured by dual luciferase reporter gene assay (Promega, Madison, WI, USA). The relative activity of firefly luciferase was normalized to Renilla luciferase [16].

\section{Flow Cytometry}

ECs at logarithmic growth were detached upon $80 \%$ confluence and seeded into 6 -well plates at $5 \times 10^{4}$ cells/well. At $72 \mathrm{~h}$ post cultivation, trypsinized ECs were centrifuged at $1000 \mathrm{r} / \mathrm{min}$ and resuspended in pre-chilled PBS. ECs were centrifuged once again and suspended in $5 \mathrm{~mL}$ binding buffer, followed by staining with $5 \mu \mathrm{L}$ annexin $\mathrm{V}$-fluorescein isothiocyanate and propidium iodide (PI) solution. ECs were then detected by a flow cytometer (BD Biosciences, Franklin Lakes, USA) to calculate the apoptosis rate.

When determining cell cycle distribution, ECs were pretreated as described above. Then, the detached ECs were fixed with $70 \%$ ethanol and incubated with $20 \mathrm{mg} / \mathrm{L}$ RNaseA (prepared by PBS with $0.2 \%$ Triton X-100) and with $50 \mathrm{mg} / \mathrm{L}$ PI. Cell cycle distribution was analyzed by the ModFit LT software (Becton, Dickinson and Company, NJ, USA). 
A

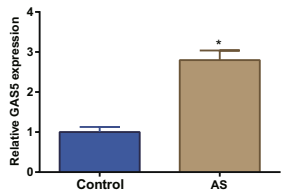

D

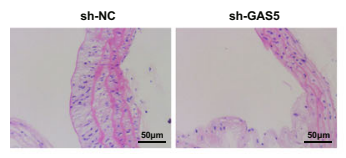

B

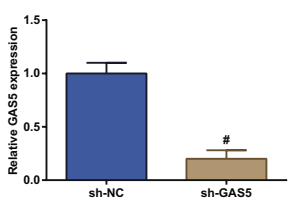

E
C

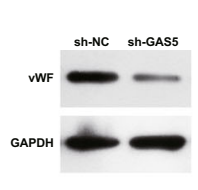

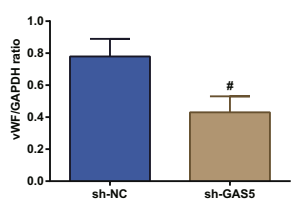

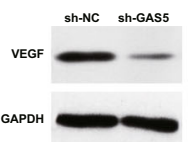

$\mathbf{F}$
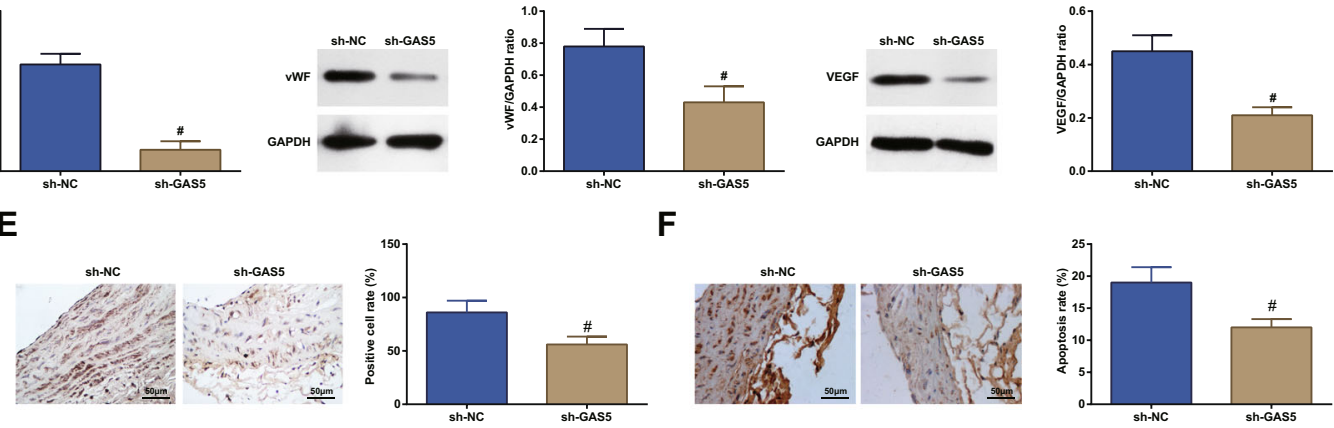

G

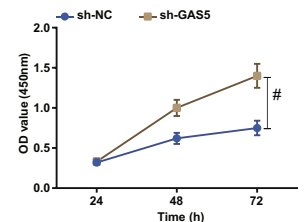

H

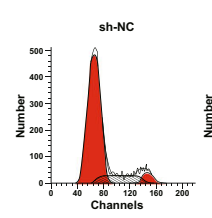

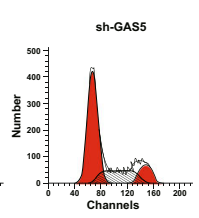

Fig. 2 Depleting GAS5 enhances proliferation and depresses apoptosis of ECs in AS. a and b RT-qPCR detected GAS5 expression in ECs. $\mathbf{c}$ Western blot assay detected vWF and VEGF protein expression in ECs. d $H \& E$ staining observed the morphology of ECs in coronary vascular tissues. e Immunohistochemistry detected VEGF expression in ECs. $\mathbf{f}$ TUNEL staining detected the apoptosis of ECs. g CCK- 8 assay detected

\section{Statistical Analysis}

All data were statistically analyzed by the SPSS21.0 (IBM, NY, USA) statistical software. The measurement data were
I

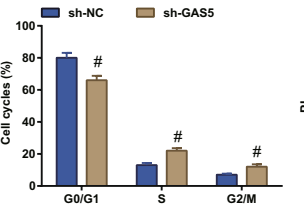

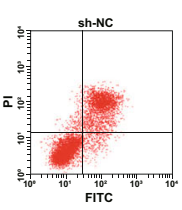
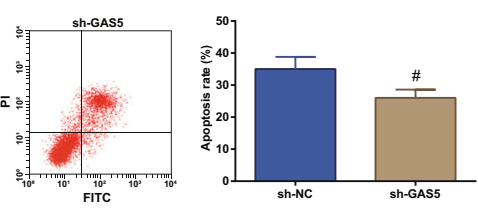

the proliferation of ECs. $\mathbf{h}$ and $\mathbf{i}$ Flow cytometry detected cell cycle distribution and apoptosis of ECs. $* P<0.05$ compared with the control group; $\# P<0.05$ compared with the sh-NC group; the measurement data were expressed as the mean \pm standard deviation. Paired $t$ test was applied for discrepancy between the two groups. $N=3$

expressed as the mean \pm standard deviation. Independent sample $t$ test was applied for comparing the discrepancy between two groups. With $P<0.05$, statistical significance was established.

Fig. 3 GAS5 targets miR-194-3p. a Jefferson website predicted the binding site between GAS5 and miR-194-3p. b Dual luciferase reporter gene assay verified the targeting relation between GAS5 and miR-194-3p. c RIP assay tested the relative enrichment of GAS5 and miR-194-3p. d RTqPCR detected miR-194-3p expression after GAS5 inhibition. \# $P<0.05$ compared with the sh$\mathrm{NC}$ group; the measurement data were expressed as the mean \pm standard deviation. Paired $t$ test was applied for discrepancy between the two groups. $N=3$
A

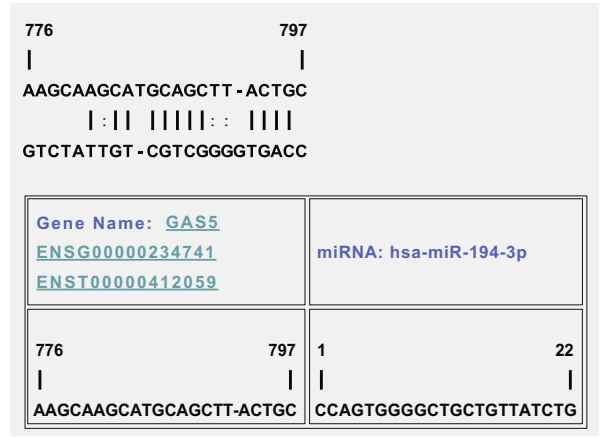

C

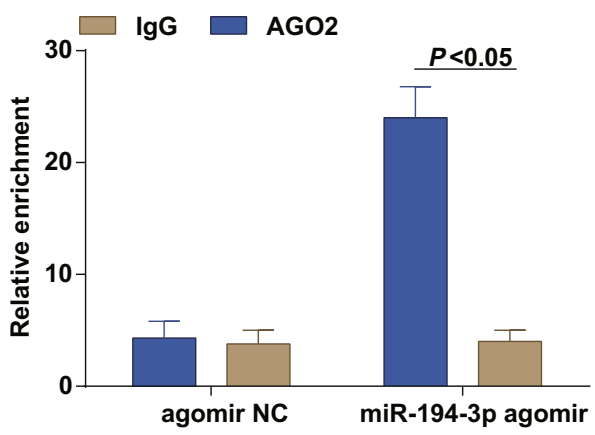

B agomir NC $\square$ miR-194-3p agomir

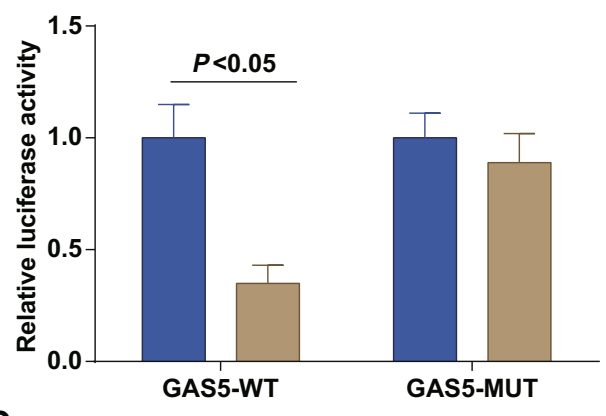

D

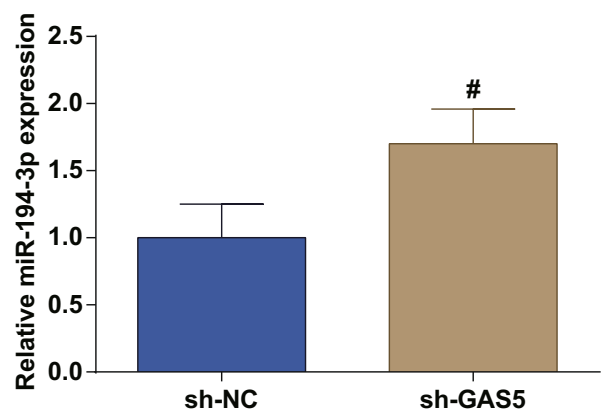




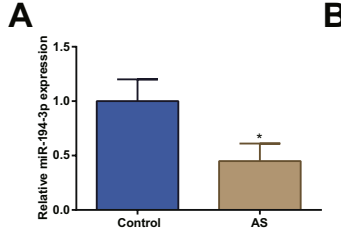

D

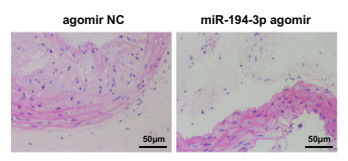

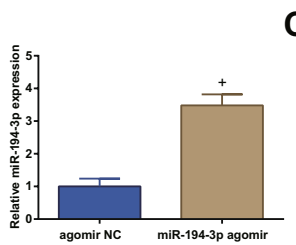

E

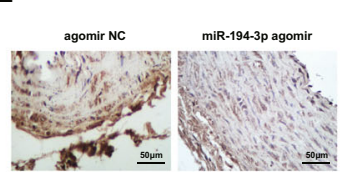

C
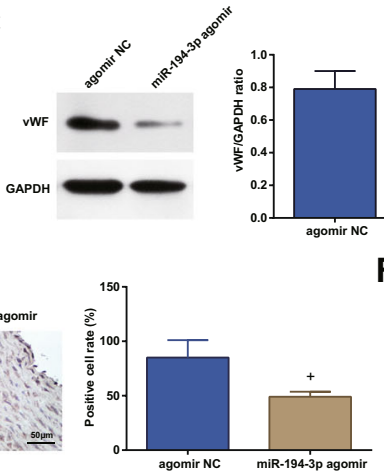

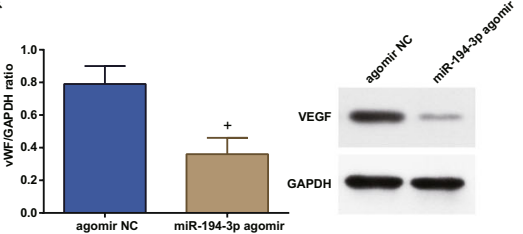

$\mathbf{F}$

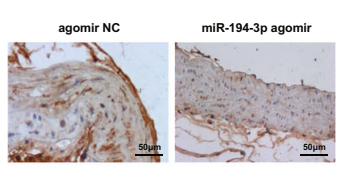

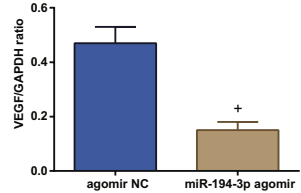

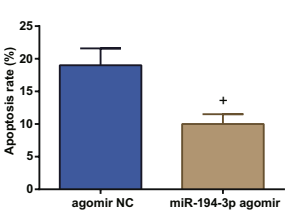

G
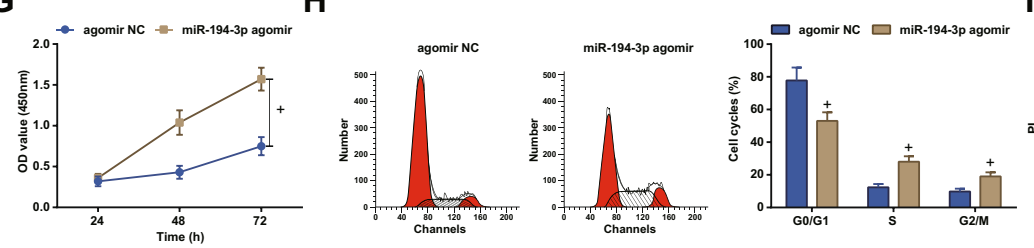

1
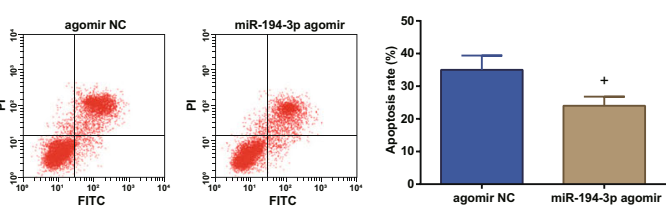

Fig. 4 Restoring miR-194-3p reinforces proliferation and impedes apoptosis of ECs in AS. a and b RT-qPCR detected miR-194-3p expression in ECs. $\mathbf{c}$ Western blot assay detected vWF and VEGF protein expression in coronary vascular tissues. $\mathbf{d} \mathrm{H} \& \mathrm{E}$ staining observed the morphology of ECs in coronary vascular tissues. e Immunohistochemistry detected VEGF expression in ECs. $\mathbf{f}$ TUNEL staining detected apoptosis of ECs.

\section{Results}

\section{Increased vWF and VEGF, Inhibited Proliferation of ECs, and Promoted Apoptosis Are Found in AS}

AS-modeled rats were established by vitamin D3 injection and high-fat diet. Then, coronary vascular tissues and ECs were collected for subsequent experiments.

Related studies have evidenced that high expression of vWF and VEGF deteriorates AS, and vWF participates in the formation of thrombosis [23, 24]. Concerning to that, vWF and VEGF g CCK-8 assay detected proliferation of ECs. $\mathbf{h}$ and $\mathbf{i}$ Flow cytometry detected cell cycle distribution and apoptosis of ECs. $* P<0.05$ compared with the control group; $+P<0.05$ compared with the agomir NC group; the measurement data were expressed as the mean \pm standard deviation. Paired $t$ test was applied for discrepancy between the two groups. $N=3$

protein expression in coronary vascular tissues were measured, and the results found that they were both upregulated in AS (Fig. 1a). Hematoxylin-eosin (H\&E) staining, immunohistochemistry, and TUNEL staining (Fig. 1b-d) detected that endothelial tissue structure was disorder, atherosclerotic plaques and thrombosis were obvious, and VEGF positive rate and apoptosis rate of ECs were highlighted in AS rats.

Based on the fact that vWF is a glycoprotein specifically secreted by ECs, which is stored in the Weibel-Palade body and possessed the capacity to identify ECs [25], immunohistochemistry was applied to identify vWF (Fig. 1e). It was
A

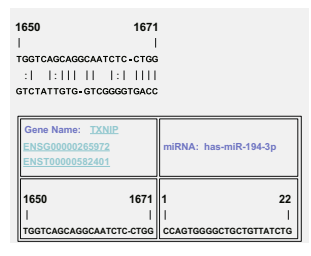

B

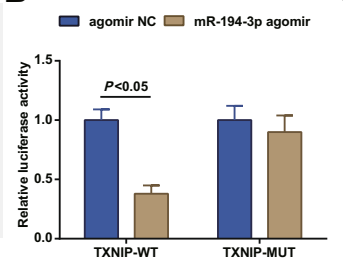

C

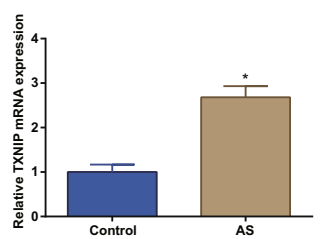

D
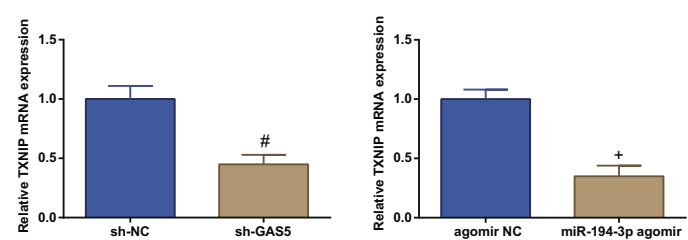

E

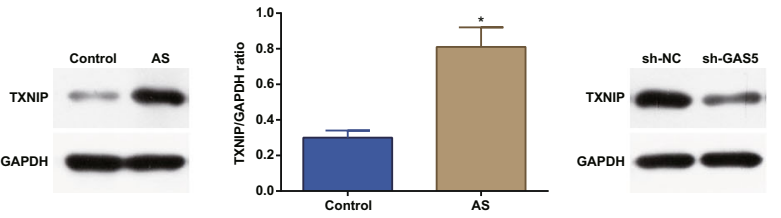

Fig. 5 GAS5 regulates TXNIP through miR-194-3p. a Jefferson predicted the binding site between miR-194-3p and TXNIP. b Dual luciferase reporter gene assay verifies the targeting relation between miR194-3p and TXNIP. $\mathbf{c}$ and $\mathbf{d}$ RT-qPCR detected TXNIP expression. e Western blot assay detected TXNIP protein expression. $* P<0.05$
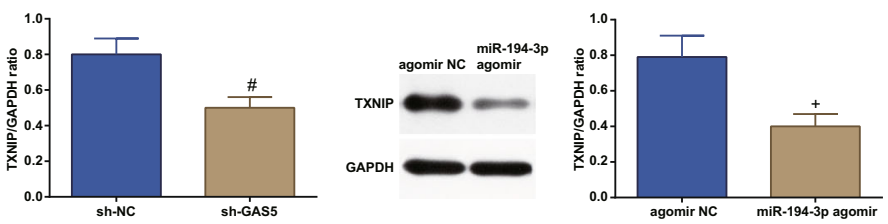

compared with the control group; \# $P<0.05$ compared with the sh-NC group; $+P<0.05$ compared with the agomir NC group; the measurement data were expressed as the mean \pm standard deviation. Paired $t$ test was applied for discrepancy between the two groups. $N=3$ 
demonstrated that the collected cells showed brownish-yellow cytoplasm, dense nucleus, and clear cell outline. Thus, those cells were confirmed as ECs. CCK-8 assay and flow cytometry (Fig. 1f-h) depicted that impaired proliferation of ECs, increased G0/ G1 cell phase, shortened $S$ and $\mathrm{G}_{2} / \mathrm{M}$ cell phases, and enhanced cell apoptosis were seen in AS rats. Those findings proved that AS was successfully modeled in rats.

\section{Depleting GAS5 Enhances Proliferation and Depresses Apoptosis of ECs in AS}

The existing literature has indicated that GAS5 knockout prevents the progression of AS [6]. Detected by RT-qPCR, it was displayed that GAS5 was overexpressed in ECs from AS rats (Fig. 2a).

GAS5 intervention assay was conducted to explore the impacts of GAS5 on coronary vascular tissues and ECs. RTqPCR presented that (Fig. 2b) GAS5 interference plasmid reduced GAS5 expression in ECs, suggesting successful downregulation of GAS5. As a result of GAS5 downregulation in ECs, VWF and VEGF protein expression were inhibited, the arrangement of ECs was improved, VEGF positive rate and apoptosis rate were decreased, proliferation of ECs was increased, cells in G0/G1 phase were reduced, and cells in $\mathrm{S}$ and $\mathrm{G}_{2} / \mathrm{M}$ phases were increased (Fig. 2c-i).

\section{GAS5 Targets miR-194-3p}

miR-194-3p has been implicated to involve in the occurrence of coronary heart disease [26]. The regulatory mechanism between GAS5 and miR-194-3p was firstly decoded by predicting their binding site through Jefferson website (Fig. $3 a)$, and subsequently validated by dual luciferase reporter gene assay (Fig. 3b). GAS5-WT/miR-194-3p-agomir transfection diminished the luciferase activity of cells while GAS5-MUT/miR-194-3p-agomir transfection caused no difference in the luciferase activity. Anti-AGO2 RIP was performed in ECs which had transfected with miR-194-3pagomir or agomir-NC (Fig. 3c). GAS5 and miR-194-3p were enriched in the AGO2. As manifested by RT-qPCR (Fig. 3d), miR-194-3p expression was elevated after GAS5 inhibition.

\section{Restoring miR-194-3p Reinforces Proliferation and Impedes Apoptosis of ECs in AS}

The involvement of miR-194-3p in the growth of ECs of coronary vascular tissues was disclosed. As detected, miR194-3p was lowly expressed in ECs in AS (Fig. 4a, b). In this study, transfection of miR-194-3p agomir elevated miR-194$3 p$ expression, hinting the successful upregulation of miR194-3p in ECs.

Functionally, miR-194-3p agomir in ECs was found to decrease VWF and VEGF protein expression and VEGF positive rate, attenuate structural damage of coronary vascular tissues, suppress apoptosis rate, reinforce cell proliferation, reduce cells in the G0/G1 phase, and increase cells in the other two phases (Fig. 4c-i).

\section{GAS5 Regulates TXNIP Through miR-194-3p}

A relevant research has pointed out that TXNIP expression is higher in AS while TXNIP knockout could attenuate AS [13]. To further investigate the regulatory mechanism downstream of miR-194-3p, Jefferson website predicted the binding site between miR-194-3p and TXNIP (Fig. 5a) while dual luciferase reporter gene assay validated the targeting relation between those two factors (Fig. 5b). Experimentally, miR-194$3 p$ agomir destroyed the luciferase activity of TXNIP-WT in cells while it had no impact on that of TXNIP-MUT. Given that, it was speculated that miR-194-3p could directly inhibit TXNIP.

To clarify that whether GAS5 regulated TXNIP via miR194-3p, RT-qPCR and western blot assay were implemented to test TXNIP expression in ECs (Fig. 5c). Clearly, TXNIP expression reached a high level in AS. Furthermore, TXNIP expression was downregulated after intervening GAS5 or upregulating miR-194-3p (Fig. 5d, e). The above results indicated that GAS5 regulated TXNIP via miR-194-3p.

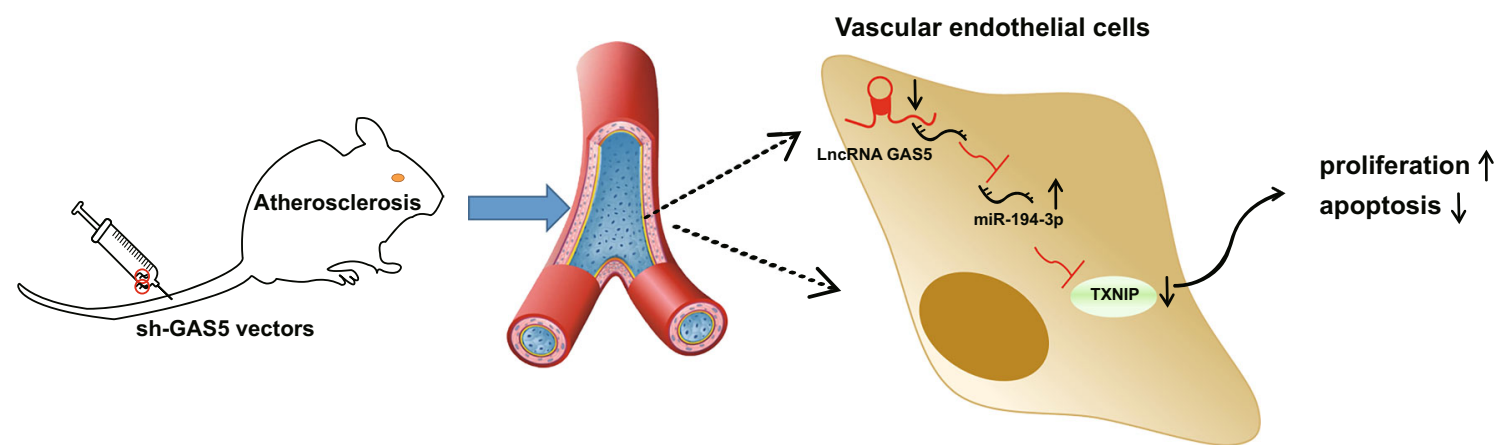

Fig. 6 Schematic diagram. Interfering with GAS5 inhibits the adsorption of GAS5 on miR-194-3p. Upregulating miR-194-3p regulates TXNIP expression, thereby protecting ECs, and reducing the formation of atherosclerotic plaques in AS 


\section{Discussion}

AS is a common cardiovascular disease, predominantly contributing to heart attack and stroke [27]. Studies have more or less mentioned the independent role of GAS5, miR-194-3p, and TXNIP in this disease, but limited to their combined reciprocal. This study has recognized the effects of GAS5/miR194-3p/TXNIP axis in AS and extracted the summary that depleted GAS5 upregulated miR-194-3p to inhibit TXNIP, thereby to promote growth of ECs and reduce the formation of atherosclerotic plaques in AS (Fig. 6).

Firstly, we tested GAS5 expression and found that it was upregulated in ECs of AS rats. Next, to decode the mechanism of GAS5 in the growth of ECs, ECs were interfered with GAS5, and then it was found that vWF and VEGF contents were reduced, apoptosis was restrained, G0/G1 cells increased, and proliferation ability of ECs was strengthened. Commonly, studies have evidenced that GAS5 is highly expressed in AS patients and modeled rats [28, 29]. Explored by a late work, GAS5 expression is manifested with an increment in AS, which promotes the progression of AS in vivo [6]. Supplemental to the finding, another research has pictured that raised GAS5 expression is obviously demonstrated in AS-modeled mice and ablating GAS5 disturbs the progression of AS [8]. In addition to that, AS patients and oxidized low-density lipoprotein-treated human aortic ECs both express GAS5 at a high level, and GAS5 depletion disrupts the apoptosis of human aortic ECs [7]. Also, a creative work has reported the elevated GAS5 expression in atherosclerotic plaques, and silencing GAS5 would interfere the apoptosis of ECs via exosomes [30].

To proceed, the molecular regulation of GAS5 on its binding genes revealed that GAS5 can specifically target miR-194$3 p$. Then, to elucidate the concrete functions of miR-194-3p in AS, miR-194-3p upregulation was projected on ECs to discover that restored miR-194-3p functioned as similar as depleted GAS5 in ECs in AS. At present, nearly no research has recorded the binding relation between GAS5 and miR-194$3 p$. It has been elaborated that miR-194 ablation is negatively connected with the growth of monocyte cells THP-1 in an intracellular inflammatory model [26]. Echoed with the present work, another work has documented that miR-194-3p expression is lower in cigarette-inflamed bronchial epithelium, and overexpressing miR-194-3p hinders apoptosis of human bronchial ECs [31]. Similarly, the reduction is noticeable in miR-194-1 expression in rats having experienced stroke [32].

Subsequently, the downstream regulatory mechanism of miR-194-3p was predicted and verified. The results manifested that miR-194-3p targeted and mediated TXNIP. Moreover, the upregulated TXNIP in AS was repressed by miR-194-3p upregulation or GAS5 downregulation, hinting that GAS5 mediated TXNIP through miR-194-3p. There is a lack of sufficient researches to identify the targeting relation between miR-194-3p and TXNIP, which needs more experiments for confirmation. TXNIP displays an increment in its expression in AS [33]. In compatibility with the finding, elevated TXNIP2 mRNA expression is characterized in acute myocardial infarction patients versus to the normal controls [34]. Besides, TXNIP expression is heightened in AS and silencing it diminishes vascular smooth muscle cell inflammation and attenuates AS [13]. Concretely, a documented report has stressed out that TXNIP expression is elevated in ECs of human carotid plaques, resulting in cell senescence [35].

\section{Conclusion}

To sum up, this research has worked out that GAS5 ablation or miR-194-3p restoration restrains TXNIP to enhance the growth of ECs in AS, exerting a referential base for developing therapeutic agents for AS. For confirmation and further outspread of the results summarized in this work, much researches are required to conduct in a scientific and logical manner.

Acknowledgements We would like to acknowledge the reviewers for their helpful comments on this paper.

Author Contribution Guobin Miao and Lizhong Wang contributed to study design; Yanbing Li contributed to manuscript editing; Yu Geng, Boda Zhou, Xuejiao Wu, Ou Zhang, Xiaonan Guan, and Yajun Xue contributed to experimental studies; Siyuan Li, Xianjing Zhuang, Jie Zhou, and Meng Chang contributed to data analysis.

Funding This work was supported by The National Natural Science Foundation of China (81970299) and High-level Health Technical Talents Training Project in Beijing Health System (12015B2003).

Availability of Data and Material Not applicable.

\section{Declarations}

Ethics Approval and Consent to Participate The experimental operation was endorsed by the ethics committee of Beijing Tsinghua Changgung Hospital (ethical number: 201920629).

Consent for Publication Not applicable.

Conflict of Interest The authors declare no competing interests.

Open Access This article is licensed under a Creative Commons Attribution 4.0 International License, which permits use, sharing, adaptation, distribution and reproduction in any medium or format, as long as you give appropriate credit to the original author(s) and the source, provide a link to the Creative Commons licence, and indicate if changes were 
made. The images or other third party material in this article are included in the article's Creative Commons licence, unless indicated otherwise in a credit line to the material. If material is not included in the article's Creative Commons licence and your intended use is not permitted by statutory regulation or exceeds the permitted use, you will need to obtain permission directly from the copyright holder. To view a copy of this licence, visit http://creativecommons.org/licenses/by/4.0/.

\section{References}

1. Taleb S (2016) Inflammation in atherosclerosis. Arch Cardiovasc Dis 109(12):708-715

2. Chiva-Blanch G et al (2020) 5-cis-, trans- and total lycopene plasma concentrations inversely relate to atherosclerotic plaque burden in newly diagnosed type 2 diabetes subjects. Nutrients 12(6)

3. Moore KJ, Sheedy FJ, Fisher EA (2013) Macrophages in atherosclerosis: a dynamic balance. Nat Rev Immunol 13(10):709-721

4. Gimbrone MA Jr (2016) and G. Garcia-Cardena, Endothelial cell dysfunction and the pathobiology of atherosclerosis. Circ Res 118(4):620-636

5. Salemi M, Cannarella R, Condorelli RA, Cimino L, Ridolfo F, Giurato G, Romano C, la Vignera S et al (2019) Evidence for long noncoding RNA GAS5 up-regulation in patients with Klinefelter syndrome. BMC Med Genet 20(1):4

6. Meng XD, Yao HH, Wang LM, Yu M, Shi S, Yuan ZX, Liu J (2020) Knockdown of GAS5 inhibits atherosclerosis progression via reducing EZH2-mediated ABCA1 transcription in ApoE(-/-) mice. Mol Ther Nucleic Acids 19:84-96

7. Liang W, Fan T, Liu L, Zhang L (2019) Knockdown of growtharrest specific transcript 5 restores oxidized low-density lipoprotein-induced impaired autophagy flux via upregulating miR-26a in human endothelial cells. Eur J Pharmacol 843:154-161

8. Shen S, Zheng X, Zhu Z, Zhao S, Zhou Q, Song Z, Wang G, Wang $Z$ (2019) Silencing of GAS5 represses the malignant progression of atherosclerosis through upregulation of miR-135a. Biomed Pharmacother 118:109302

9. Feinberg MW, Moore KJ (2016) MicroRNA regulation of atherosclerosis. Circ Res 118(4):703-720

10. Qu S, Yang L, Liu Z (2020) MicroRNA-194 reduces inflammatory response and human dermal microvascular endothelial cells permeability through suppression of TGF-beta/SMAD pathway by inhibiting THBS1 in chronic idiopathic urticaria. J Cell Biochem 121(1):111-124

11. Brumm AJ, Nunez S, Doroudchi MM, Kawaguchi R, Duan J, Pellegrini M, Lam L, Carmichael ST et al (2017) Astrocytes can adopt endothelial cell fates in a p53-dependent manner. Mol Neurobiol 54(6):4584-4596

12. Yang $Y$ et al (2019) MicroRNA26a inhibits cisplatin-induced renal tubular epithelial cells apoptosis through suppressing the expression of transient receptor potential channel 6 mediated dynamin-related protein 1. Cell Biochem Funct

13. Byon $\mathrm{CH}$, Han T, Wu J, Hui ST (2015) Txnip ablation reduces vascular smooth muscle cell inflammation and ameliorates atherosclerosis in apolipoprotein E knockout mice. Atherosclerosis 241(2):313-321

14. Chen M, Li W, Zhang Y, Yang J (2018) MicroRNA-20a protects human aortic endothelial cells from Ox-LDL-induced inflammation through targeting TLR4 and TXNIP signaling. Biomed Pharmacother 103:191-197
15. Song CL, Wang JP, Xue X, Liu N, Zhang XH, Zhao Z, Liu JG, Zhang CP et al (2017) Effect of circular ANRIL on the inflammatory response of vascular endothelial cells in a rat model of coronary atherosclerosis. Cell Physiol Biochem 42(3):1202-1212

16. Her OS, Chen YC, Yen NS (2017) Mathematical values in the processing of Chinese numeral classifiers and measure words. PLoS One 12(9):e0185047

17. Wang Y, Yang X, Jiang A, Wang W, Li J, Wen J (2019) Methylation-dependent transcriptional repression of RUNX3 by KCNQ1OT1 regulates mouse cardiac microvascular endothelial cell viability and inflammatory response following myocardial infarction. FASEB J 33(12):13145-13160

18. Huang F, Liu Y, Yang X, Che D, Qiu K, Hammock BD, Wang J, Wang MH et al (2017) Shexiang Baoxin pills promotes angiogenesis in myocardial infarction rats via up-regulation of 20-HETEmediated endothelial progenitor cells mobilization. Atherosclerosis 263:184-191

19. Livak KJ, Schmittgen TD (2001) Analysis of relative gene expression data using real-time quantitative PCR and the 2(-Delta Delta $\mathrm{C}(\mathrm{T})$ ) method. Methods 25(4):402-408

20. Song J, Kim YK (2017) Identification of the role of miR-142-5p in Alzheimer's disease by comparative bioinformatics and cellular analysis. Front Mol Neurosci 10:227

21. Grieco FA, Sebastiani G, Juan-Mateu J, Villate O, Marroqui L, Ladrière L, Tugay K, Regazzi R et al (2017) MicroRNAs miR$23 a-3 p, m i R-23 b-3 p$, and miR-149-5p regulate the expression of proapoptotic BH3-only proteins DP5 and PUMA in human pancreatic beta-cells. Diabetes 66(1):100-112

22. Chen G, Li Y, He Y, Zeng B, Yi C, Wang C, Zhang X, Zhao W et al (2020) Upregulation of circular RNA circATRNL1 to sensitize oral squamous cell carcinoma to irradiation. Mol Ther Nucleic Acids 19:961-973

23. Hamamdzic D, Fenning RS, Patel D, Mohler ER III, Orlova KA, Wright AC, Llano R, Keane MG et al (2010) Akt pathway is hypoactivated by synergistic actions of diabetes mellitus and hypercholesterolemia resulting in advanced coronary artery disease. Am J Physiol Heart Circ Physiol 299(3):H699-H706

24. Sun S, Chai S, Zhang F, Lu L (2020) Overexpressed microRNA$103 a-3 p$ inhibits acute lower-extremity deep venous thrombosis via inhibition of CXCL12. IUBMB Life 72(3):492-504

25. Varenne O, Pislaru S, Gillijns H, van Pelt N, Gerard RD, Zoldhelyi P, van de Werf F, Collen D et al (1998) Local adenovirus-mediated transfer of human endothelial nitric oxide synthase reduces luminal narrowing after coronary angioplasty in pigs. Circulation 98(9): 919-926

26. Tian H, Liu C, Zou X, Wu W, Zhang C, Yuan D (2015) MiRNA194 regulates palmitic acid-induced toll-like receptor 4 inflammatory responses in THP-1 cells. Nutrients 7(5):3483-3496

27. Amar S, Engelke M (2015) Periodontal innate immune mechanisms relevant to atherosclerosis. Mol Oral Microbiol 30(3):171-185

28. Chen L, Yao H, Hui JY, Ding SH, Fan YL, Pan YH, Chen KH, Wan JQ et al (2016) Global transcriptomic study of atherosclerosis development in rats. Gene 592(1):43-48

29. Ye J, Wang C, Wang D, Yuan H (2018) LncRBA GSA5, upregulated by ox-LDL, aggravates inflammatory response and MMP expression in THP-1 macrophages by acting like a sponge for miR-221. Exp Cell Res 369(2):348-355

30. Chen L, Yang W, Guo Y, Chen W, Zheng P, Zeng J, Tong W (2017) Exosomal lncRNA GAS5 regulates the apoptosis of macrophages and vascular endothelial cells in atherosclerosis. PLoS One 12(9): 0185406

31. Zhou T, Zhong Y, Hu Y, Sun C, Wang Y, Wang G (2018) PM2.5 downregulates miR-194-3p and accelerates apoptosis in cigarette- 
inflamed bronchial epithelium by targeting death-associated protein kinase 1. Int J Chron Obstruct Pulmon Dis 13:2339-2349

32. Takuma A et al (2017) Gene expression analysis of the effect of ischemic infarction in whole blood. Int J Mol Sci:18(11)

33. Yue LM, Gao YM, Han BH (2019) Evaluation on the effect of hydrogen sulfide on the NLRP3 signaling pathway and its involvement in the pathogenesis of atherosclerosis. J Cell Biochem 120(1): 481-492

34. Zhang Y et al (2018) Differential expression of TXNIP isoforms in the peripheral leukocytes of patients with acute myocardial infarction. Dis Markers 2018:9051481
35. Riahi Y, Kaiser N, Cohen G, Abd-Elrahman I, Blum G, Shapira OM, Koler T, Simionescu M et al (2015) Foam cell-derived 4hydroxynonenal induces endothelial cell senescence in a TXNIPdependent manner. J Cell Mol Med 19(8):1887-1899

Publisher's Note Springer Nature remains neutral with regard to jurisdictional claims in published maps and institutional affiliations. 